\title{
MACROSOMIA FETAL: PREVALENCIA, FACTORES DE RIESGO ASOCIADOS Y COMPLICACIONES EN EL HOSPITAL REGIONAL DE ICA, PERU
}

\author{
Ismael Rolando Gonzáles-Tipiana ${ }^{1, a, b}$ \\ ${ }^{1}$ Facultad de Medicina, Universidad Nacional San Luis Gonzaga. Ica, Perú. \\ ${ }^{a}$ Doctor en Salud Pública, ${ }^{b}$ Médico Pediatra

\section{RESUMEN} \\ Objetivo: Determinar la prevalencia de macrosomía fetal, identificar los factores de riesgo materno asociados y las \\ principales complicaciones de los recién nacidos macrosómicos atendidos en un hospital de la provincia de Ica, Perú. \\ Material y métodos: Estudio descriptivo, retrospectivo, transversal, incluyó a 129 recién nacidos macrosómicos, durante el \\ periodo comprendido entre el 1 de enero al 31 de diciembre del 2,002. Resultados: De 2,550 recién nacidos en el periodo de \\ estudio, $129(5 \%)$ fueron macrosómicos, la edad materna fue de 31 a 40 años en el $58 \%$, el peso habitual de 70 a $99 \mathrm{Kg}$. en \\ el $81 \%$, multiparidad $65 \%$ y embarazo prolongado $7 \%$. El $53 \%$ nació por parto vaginal y el $47 \%$ por cesárea. El sexo \\ predominante fue el femenino con el $62 \%$, el Apgar promedio entre 7 y 10 al minuto representó el $93 \%$, la edad gestacional \\ por examen físico (Test de Capurro) fue de 37 a 41 semanas en el $93 \%$ de los casos. Respecto al peso al nacimiento $99.2 \%$ \\ pesó entre 4,000 y $4,999 \mathrm{gr}, 0.8 \%$ pesó más de $5,000 \mathrm{gr}$. (Peso promedio $4,194 \mathrm{gr}$, mínimo $4,000 \mathrm{gr}$, máximo $5,180 \mathrm{gr}$ ). La \\ morbilidad neonatal representó el $30 \%$. No tuvimos mortalidad neonatal. Las principales complicaciones fueron: $5 \%$ \\ hiperbilirrubinemia, $2 \%$ hipoxia perinatal, $8 \%$ hipoglucemia y $15 \%$ trauma obstétrico. Conclusiones: La Prevalencia de \\ macrosomía fue $5 \%$, los factores maternos asociados fueron: la edad materna mayor de 30 años y el peso promedio habitual \\ de la madre mayor de $70 \mathrm{Kg}$, multiparidad y embarazo prolongado. El sexo predominante fue el femenino. No hubo diferencia \\ significativa en lo referente a la vía de terminación del parto. Las principales complicaciones fueron: traumatismo obstétrico \\ (cefalohematoma, caput sucedaneum y fractura de clavícula), hiperbilirrubinemia e hipoglucemia.
}

Palabras clave: Macrosomía fetal, Factores de riesgo, Mortalidad Infantil. (fuente: DeCS BIREME)

\section{FETAL MACROSOMIA: PREVALENCE, RISK FACTORS ASSOCIATED AND COMPLICATIONS IN THE REGIONAL HOSPITAL IN ICA, PERU}

\begin{abstract}
Objective: To Determine the prevalence of fetal macrosomia, identify the maternal risk factors associated and the main complications of macrosomic newborns treated at a hospital in the province of Ica, Peru. Methods: Descriptive study, retrospective, cross-sectional, included 129 macrosomic newborns, during the period from January 1 to December 31 of January 2002. Results: Of 2550 newborns in the study period, 129 (5\%) were macrosomic, maternal age was 31 to 40 years in the $58 \%$, the usual weight of 70 to $99 \mathrm{~kg}$ in the $81 \%, 65 \%$ and multiparity prolonged pregnancy $7 \%$. The $53 \%$ was born by vaginal birth and 47 per cent by cesarean section. The predominant sex was female with $62 \%$, the Apgar score average between 7 and 10 to minute represented $93 \%$, the gestational age by physical exam (Test of Capurro) was 37 to 41 weeks in $93 \%$ of the cases. With regard to birth weight $99.2 \%$ weight between 4.000 and $4.999 \mathrm{gr}, 0.8 \%$ weight more than $5.000 \mathrm{gr}$. (Average Weight $4.194 \mathrm{gr}$, minimum $4.000 \mathrm{gr}$, maximum $5.180 \mathrm{gr}$ ). Neonatal morbidity represented $30 \%$. We had no neonatal mortality. The main complications were: $5 \%$ hyperbilirubinemia, $2 \%$ perinatal hypoxia, hypoglycemia, and $8 \% 15 \%$ obstetric trauma .Conclusions: The prevalence of macrosomia was $5 \%$, the associated maternal factors were: the maternal age greater than 30 years and the average weight of the usual mother greater than $70 \mathrm{Kg}$, multiparity and prolonged pregnancy. The predominant sex was female. There was no significant difference in regard to route of completion of the birth. The main complications were: obstetric trauma (cephalohematoma, caput sucedaneum and clavicle fracture), hyperbilirubinemia and hypoglycemia.
\end{abstract}

Key words: Fetal Macrosomía, Risk factors, Infant Mortality. (source: MeSH NLM)

\section{INTRODUCCIÓN}

Macrosomía es el término utilizado para describir a un niño recién nacido demasiado grande. Aún no se ha llegado a la conclusión sobre el peso límite para definir a un niño macrosómico. Algunos autores sugieren un peso mayor de 4000 grs, mientras que otros proponen un peso superior a 4100 ó $4500 \mathrm{grs}^{(1,3)}$. La macrosomía puede estar asociada con muchos factores de riesgo recogidos en los antecedentes de la paciente antes y durante el embarazo. Entre ellas tenemos el antecedente de peso materno antes de la concepción mayor a $90 \mathrm{Kg}$, multiparidad, embarazo prolongado, macrosomía en embarazo previo, sexo mascu- lino, macrosomía de la madre al nacer, diabetes materna, entre otros ${ }^{(2,4,5)}$. Entre los principales problemas neonatales de recién nacido macrosómico se describe el traumatismo obstétrico (equimosis, cefalohematoma, injuria del plexo braquial, fracturas de clavícula, parálisis del diafragma, etc. Los trastornos metabólicos encontrados son la hipoglucemia debido a hiper insulinemia por pobre ingesta calórica. Otro de los problemas asociados es la policitemia que condiciona hiperviscosidad y consecuentemente hipoglucemia, trombosis, hipervolemia e ictericia. Aquellos macrosómicos hijos de madres diabéticas se asocian con 
malformaciones congénitas, deficiencia de surfactante y síndrome de aspiración meconial.

Es difícil predecir la macrosomía fetal, ya que en ocasiones el estimado clínico y el ultrasonido (circunferencia cefálica, torácica y abdominal) del peso fetal están propensos a presentar errores. ${ }^{(3)}$ Teniendo en cuenta que los fetos de excesivo peso aumentan la morbimortalidad materno fetal y que muchos autores plantean una pérdida perinatal de alrededor del $7,2 \%$ y que el parto es la vía que constituye el punto neurálgico en el pronóstico de esta, decidimos realizar un estudio en nuestro medio de los recién nacidos macrosómicos y realizar una identificación de la prevalencia, factores de riesgo materno asociados y complicaciones en los recién nacidos ${ }^{(1,3)}$.

\section{MATERIALES Y MÉTODOS}

Se realizó una investigación de tipo descriptiva, retrospectivo y transversal del comportamiento de la macrosomía fetal en el Servicio Neonatología del Hospital Regional de Ica-Perú, durante el período comprendido entre el $1^{\circ}$ de Enero al 31 de Diciembre de 2002. Durante este periodo nacieron 2550 niños, de los cuales 129 pesaron 4000 gr ó más.

Los datos fueron tomados de las historias clínicas maternas y de los recién nacidos y fueron seleccionadas variables como: antecedentes maternos, edad y peso habitual de la madre, multiparidad, vía de terminación del parto, Apgar al nacer y morbimortalidad del recién nacido, entre otros. Para probar la consistencia y confiabilidad del instrumento elaborado se utilizó una prueba piloto en 35 recién nacidos macrosómicos del Hospital Regional de lca y se procesaron estadísticamente usando el programa Epi Info 2000; asimismo se realizó un análisis univariado y bivariado con IC 95\% considerando un $p<0,05$ como estadísticamente significativo.

Finalmente se confeccionaron tablas, para facilitar el análisis y discusión de los resultados. Dentro de nuestros objetivos se encuentran el determinar la prevalencia de macrosomía; identificar los antecedentes maternos más frecuentes; valorar la vía del parto más frecuente y evaluar el Apgar, sexo y morbimortalidad del recién nacido macrosómico.

Tabla 2. Complicaciones en $\mathrm{RN}$ macrosomicos

\begin{tabular}{lccc}
\hline \multicolumn{1}{c}{ Variables } & $\mathbf{n = 1 2 9}$ & $\mathbf{\%}$ & $\mathbf{P}$ \\
\hline Trauma obstétrico & 19 & 15 & \\
Cefalohematoma & 4 & 2 & $\mathbf{0 , 4 4}$ \\
Capul sucedaneum & 10 & 8 & \\
Fractura de clavícula & 5 & 5 & \\
Hipoglicemia & 10 & 8 & $\mathbf{0 , 6 3}$ \\
Hiperbilimrubinemia & 6 & 5 & $\mathbf{0 , 5 8}$ \\
Hipoxia perinatal & 3 & 2 & $\mathbf{0 , 4 7}$ \\
\hline
\end{tabular}

\section{RESULTADOS}

El tipo parto más frecuente fue el eutócico (53\%) y cesárea el $(47 \%)$, predominó el sexo femenino con el $(62 \%)$ y el masculino represento el (38\%). Los recién nacidos presentaron depresión respiratoria severa, Apgar entre 0 -3 minuto (2\%), depresión respiratoria moderada Apgar entre $4-6$ a los minuto (5\%) y Apgar $7-10$ al minuto (93\%). (Tabla 1). La morbilidad neonatal fue $(30 \%)$ y con más frecuencia el trauma obstétrico (caput sucedaneum, cefalohematoma y fractura de clavícula), asimismo se asociaron, hipoglucemia, hiperbilirrubinemia e hipoxia perinatal (Tabla 2). No tuvimos mortalidad neonatal.

Tabla 1. Factores de riesgo asociados a macrosomía fetal

\begin{tabular}{lccc}
\hline \multicolumn{1}{c}{ Variables } & $\mathbf{n = 1 2 9}$ & $\mathbf{\%}$ & $\mathbf{p}$ \\
\hline Edad Materna & & & \\
10-19 años & 8 & 6 & \\
20-30 años & 42 & 33 & $\mathbf{0 , 4 9}$ \\
$31-40$ años & 75 & 58 & \\
> 40 años & 4 & 3 & \\
Peso habitual materno & & & \\
50-59 Kg & 24 & 19 & $\mathbf{0 , 5 3}$ \\
70-99 Kg & 105 & 81 & \\
Paridad & & & \\
Primípara & 32 & 25 & \\
Multípara & 84 & 65 & $\mathbf{0 , 4 6}$ \\
Gran multípara & 13 & 10 & \\
Edad Gestacional & & & \\
37-41 semanas & 120 & 93 & $\mathbf{0 , 3 2}$ \\
$>$ 42 semanas & 9 & 7 & \\
Tipo de parto & & & \\
Eutócico & 68 & 53 & $\mathbf{0 , 4 1}$ \\
Cesárea & 61 & 47 & \\
Sexo & & & \\
Masculino & 80 & 62 & $\mathbf{0 , 5 0}$ \\
Femenino & 49 & 38 & \\
Apgar & & & \\
0-3 & 2 & 2 & $\mathbf{0 , 1 8}$ \\
4-6 & 7 & 5 & \\
7-10 & 120 & 93 & \\
\hline
\end{tabular}

\section{DISCUSIÓN}

La macrosomía fetal mostró una prevalencia promedio de $5 \%$. Su diagnóstico y estimado clínico y por ultrasonido del peso fetal (diámetro biparietal, longitud del fémur $y$ circunferencia abdominal) están propensos a error. ${ }^{(3)}$ Zamorski plantea que sólo el $50 \%$ de los bebés determinados como macrosómicos, al nacer fueron bien diagnosticados (3). Essel JK, identificó al embarazo prolongado, multiparidad, peso materno mayor a $70 \mathrm{Kg}$ ò más y gestantes en su tercera década como factores de riesgo materno, asociados a macrosomía ${ }^{(9)}$. Es de señalar que en nuestro trabajo los factores de riesgo asociados a macrosomía (Tabla 1), fueron edad materna mayor a 30 años, embarazo prolongado, obesidad y multiparidad, sobresalieron con $(41,5 \%)$, no se encontró ninguna gestante diabética y no presentaron factores de riesgo asociados el $(58,5 \%)$ muy similar a lo revisado en la 
literatura, por lo que cobra gran importancia. Cuando existe diabetes, embarazo prolongado y obesidad, el riesgo de macrosomía fetal oscila entre el 5 - $15 \%$ (Cunnigham, 1998). ${ }^{(1)}$ No obstante, Lepercq encontró que el $80 \%$ de los bebés macrosómicos son nacidos de madres no diabéticas.

(2) La obesidad materna y el aumento de peso durante el embarazo, tienen impacto directo sobre el peso del niño al nacer y las complicaciones maternas, al igual que la edad avanzada y la alta paridad de las madres ${ }^{(4,6)}$.

Existen discrepancias entre los distintos autores en relación con la vía del parto. Gaven plantea que una política de parto por cesárea electiva en casos de sospecha de macrosomía fetal, tuvo un efecto insignificante sobre la prevalencia del daño del plexo braquial y aumentó considerablemente la tasa de cesárea y de morbilidad quirúrgica ${ }^{(1)}$. Otros, como Wollschlaeger, sugieren que la distocia de hombros y el daño del plexo braquial son eventos impredecibles y que las intervenciones planificadas (cesáreas) basadas en el estimado del peso, no reducen la incidencia de distocia de hombros ni disminuyen las consecuencias atribuibles a la macrosomía ${ }^{(4)}$. Moconu plantea que la alta tasa de parto vaginal y la baja incidencia de distocias de hombros pueden fundamentar la no realización de cesáreas electivas para el parto en los infantes macrosómicos. ${ }^{(6)}$ Sin embargo, Parry plantea que aún en neonatos no macrosómicos el diagnóstico de sospecha de macrosomía fetal está asociado con un incremento significativo de la tasa de cesárea ${ }^{(7)}$.

En caso de existir complicaciones del embarazo como gestosis, diabetes, etc., hay razones para inducción electiva o terminar el embarazo si el cálculo del peso está alrededor de los $4000 \mathrm{gr}$, sin embargo cada servicio debe tener una conducta para cada caso en particular. El embarazo complicado por macrosomía fetal es mejor manejarlo con conducta expectante y cuando el trabajo de parto falla en su progreso, la posibilidad de desproporción cefalopélvica debe ser considerada dentro del contexto de un mejor estimado del peso fetal ${ }^{(3)}$.

En nuestro trabajo predominó el parto eutócico (53\%) y cesáreas el $(47 \%)$. La morbilidad fetal fue $(30 \%)$, con predominio del traumatismo obstétrico, cefalohematoma caput sucedaneum y fractura de clavícula en los partos eutócicos.

Al analizar los partos ocurridos por cesárea se evidencia un número menor de complicaciones fetales (trauma obstétrico) que lógicamente facilitan la no producción de estos, por una mayor facilidad de la extracción del feto no sometido al canal del parto. Sin embargo, si consideramos los riesgos maternos conocidos en la morbimortalidad materna por cesáreas, hay que analizar la conveniencia o no de su realización. En relación al Apgar de los recién nacidos, el (93\%) tuvieron Apgar entre 7-10, (5\%) entre 4-6 y $(2 \%)$ entre $0-3$. Se presentaron 2 casos asfixia grave. Predominó, el sexo femenino no coincidiendo con la bibliografía revisada, así como el líquido amniótico claro.

Podemos concluir que la prevalencia de macrosomía fetal es del $5 \%$. Los factores de riesgo asociados encontrados más frecuentemente en la macrosomía fetal fueron: edad materna mayor a 30 años, peso materno previo embarazo entre 70 a $90 \mathrm{Kg}$, embarazo prolongado y multiparidad. No se encontró ninguna gestante diabética. Existió predominio del parto eutócico, La morbilidad neonatal fue significativa (30\%). Predominó el sexo femenino y el Apgar de la mayoría de recién nacidos fue adecuado. Se presentaron 2 casos de asfixia grave. No tuvimos mortalidad neonatal.

Financiamiento: El estudio fue autofinanciado.

Conflictos de interés: El autor declara no tener conflictos de interés en la publicación de este artículo.

\section{Correspondencia:}

Ismael Rolando Gonzáles Tipiana

Correo electrónico: ismael_w2002@yahoo.com

\section{REFERENCIAS BIBLIOGRÁFICAS}

1. Cunnigham G, Mac Donald $\mathbf{P}$, Gant $\mathbf{N}$, Leveno K, Gilstrop LC. Macrosomía fetal. Williams Obstetricia. 20 ed. Buenos Aires: Editorial Médica Panamericana, 1998: 797-801.

2. Lepercg J, Timsit J, Hanguel-de Monzón S. Etiopathog of fetal macrosomía. J Gynecol Obstet Biol Reprod 2000; 29 (1):6-12.

3. Zamorski MA, Biggs WS. Management of suspected fetal macrosomía. Am Fam Phis 2001; 63(2):302-6.

4. Haram K, Bergsjo P, Pironem J. Suspected large fetus in the last period of pregnancy. A difficult problem. Tidss Kr Nor Large Foren 2001; 121 (11):1369-73.

5. Dang K, Homko C, Reece EA. Factors associated with fetal macrosomía in offspring of gestacional diabetic woman. J Matern Fetal Med 2000; 9(2):114-7.

6. Mocanu EU, Greene RA, Byrne BM, Turner MJ. Obstetric and neonatal outcome of babies weighing more than $4.5 \mathrm{~kg}$ an analysis by parity. Eur $\mathrm{J}$ Obstet Gynecol Reprod Biol 2000; 92(2):229-33.

7. Parry SS, Schder HM. Ultrasographic prediction of fetal macrosomía association with cesarean delivery. J Reprod Med 2000; 45(1):17-22.

8. Essel JK, Opai Tetteh ET. Macrosomía fetal y factores de riesgo materno. S. Agr-Med-J. 1995; 85(1): 43-6

Recibido: 15/03/2012

Aceptado para publicación: 13/07/2012 